\title{
Serum Neurofilament Light Chain Levels Are Related to Small Vessel Disease Burden
}

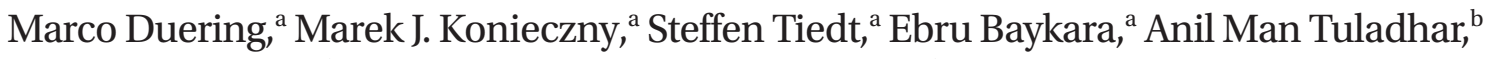 \\ Esther van Leijsen, ${ }^{\mathrm{b}}$ Philippe Lyrer, ${ }^{\mathrm{c}}$ Stefan T. Engelter, ${ }^{\mathrm{c}, \mathrm{d}}$ Benno Gesierich, ${ }^{\mathrm{a}}$ Melanie Achmüller, ${ }^{\mathrm{a}}$ \\ Christian Barro, ${ }^{\mathrm{e}}$ Ruth Adam, ${ }^{\mathrm{a}}$ Michael Ewers, ${ }^{\mathrm{a}}$ Martin Dichgans, ${ }^{\mathrm{a}, \mathrm{f}}$ Jens Kuhle, ${ }^{\mathrm{e}}$ Frank-Erik de Leeuw, \\ Nils Peters ${ }^{c}$ \\ ${ }^{a}$ Institute for Stroke and Dementia Research (ISD), University Hospital, Ludwig-Maximilians-University Munich, Munich, Germany \\ ${ }^{b}$ Department of Neurology, Donders Institute for Brain, Cognition and Behaviour, Radboud University Medical Center, Nijmegen, the Netherlands \\ 'Stroke Center and Department of Neurology, University Hospital Basel, Basel, Switzerland \\ ${ }^{\mathrm{d}}$ Neurorehabilitation Unit, University of Basel and University Center for Medicine of Aging, Felix Platter Hospital, Basel, Switzerland

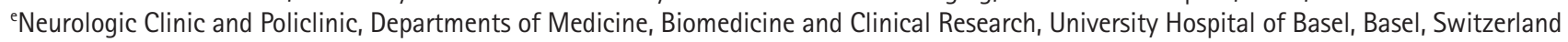 \\ ${ }^{f}$ Munich Cluster for Systems Neurology (SyNergy), Munich, Germany
}

Background and Purpose Neurofilament light chain (NfL) is a blood marker for neuroaxonal damage. We assessed the association between serum NfL and cerebral small vessel disease (SVD), which is highly prevalent in elderly individuals and a major cause of stroke and vascular cognitive impairment. Methods Using a cross-sectional design, we studied 53 and 439 patients with genetically defined SVD (Cerebral Autosomal-Dominant Arteriopathy with Subcortical Infarcts and Leukoencephalopathy [CADASIL]) and sporadic SVD, respectively, as well as 93 healthy controls. Serum NfL was measured by an ultrasensitive single-molecule array assay. We quantified magnetic resonance imaging (MRI) markers of SVD, i.e., white matter hyperintensity volume, lacune volume, brain volume, microbleed count, and mean diffusivity obtained from diffusion tensor imaging. Clinical characterization included neuropsychological testing in both SVD samples. CADASIL patients were further characterized for focal neurological deficits (National Institutes of Health stroke scale [NIHSS]) and disability (modified Rankin scale [mRS]).

Results Serum NfL levels were elevated in both SVD samples ( $P<1 \mathrm{e}-05$ compared with controls) and associated with all SVD MRI markers. The strongest association was found for mean diffusivity (CADASIL, $R^{2}=0.52, P=1.2 \mathrm{e}-09$; sporadic $\left.S V D, R^{2}=0.21, P<1 \mathrm{e}-15\right)$. Serum NfL levels were independently related to processing speed performance (CADASIL, $R^{2}=0.27, P=7.6 e-05$; sporadic $S V D, R^{2}=0.06$, $P=4.8 \mathrm{e}-08$ ), focal neurological symptoms (CADASIL, NIHSS, $P=4.2 \mathrm{e}-05$ ) and disability (CADASIL, mRS, $P=3.0 \mathrm{e}-06)$.

Conclusions We found serum NfL levels to be associated with both imaging and clinical features of SVD. Serum NfL might complement MRI markers in assessing SVD burden. Importantly, SVD needs to be considered when interpreting serum NfL levels in the context of other age-related diseases.

Keywords Biomarkers; Serum; Cerebral small vessel diseases; Magnetic resonance imaging; Dementia, vascular
Correspondence: Marco Duering Institute for Stroke and Dementia Research (ISD), University Hospital, Ludwig-Maximilians-University Munich, Feodor-Lynen-Str. 17, Munich 81377, Germany Tel: +49-89-4400-46166

Fax: +49-89-4400-46040 E-mail: marco.duering@med.uni-muenchen.de

Received: November 1, 2017 Revised: February 1, 2018 Accepted: February 2, 2018 


\section{Introduction}

Neurofilament light chain (NfL) is an emerging blood marker for neuroaxonal damage. A role of serum NfL has been established in multiple neurological diseases affecting the elderly population, such as motor neuron disease, ${ }^{1}$ Alzheimer's disease $_{1}{ }^{2}$ and frontotemporal dementia. ${ }^{3}$ However, the factors influencing serum $\mathrm{NfL}$ levels in the elderly population are not completely understood. Cerebral small vessel disease (SVD) is a highly prevalent condition and a major cause of stroke, vascular cognitive impairment, and eventually loss of independence. ${ }^{4}$ Recent studies suggest that cerebrovascular pathology has an effect on serum NfL. Elevated serum levels were observed in patients with a recent small subcortical infarct ${ }^{5}$ and in patients with stroke caused by cervical artery dissection. ${ }^{6}$ Moreover, NfL levels in the cerebrospinal fluid (CSF) correlated significantly with white matter hyperintensity (WMH) load, one of magnetic resonance imaging (MRI) markers of SVD burden.' However, a detailed account of the relationship between SVD burden and NfL in the blood is lacking.

The aim of the current study was to assess the association between serum NfL and SVD burden as assessed by MRI and clinical status. We first analyzed patients with the genetically defined SVD, Cerebral Autosomal-Dominant Arteriopathy with Subcortical Infarcts and Leukoencephalopathy (CADASIL). Because these patients already show severe SVD at a young age, confounding by other age-related pathologies impacting on serum NfL levels, such as neurodegenerative pathology, can be largely excluded. ${ }^{8}$ To validate our findings and assess their generalizability toward the more common, sporadic form of SVD, we further analyzed a large, independent sample of patients with sporadic SVD.

We hypothesized that serum NfL levels are associated with MRI markers for SVD, i.e., WMH volume, lacune volume, brain volume, microbleed count, and mean diffusivity from diffusion tensor imaging (DTI). Furthermore, we hypothesized that serum $\mathrm{NfL}$ levels are associated with clinical status in SVD, in particular cognitive performance, focal neurological deficits, and disability.

\section{Methods}

\section{Study participants}

CADASIL patients were recruited into the ongoing, prospective "VASCAMY (vascular and amyloid predictors of neurodegeneration and cognitive decline in non-demented subjects)" study. The diagnosis was confirmed by either molecular genetic testing or ultrastructural analysis of skin biopsies. Exclusion criteria were the presence or history of (1) diabetes mellitus (because of its pronounced effect on brain structure), (2) other known neurological or psychiatric diseases, and (3) clinically apparent stroke within the last 3 months. Fifty-four patients gave consent for the biobanking procedure and were therefore included in this analysis. All examinations (clinical assessment, neuropsychological testing, blood draw, and MRI) were performed on the same day or within 2 consecutive days. One subject was excluded because of missing MRI data (scanner malfunction). The final sample comprised 53 CADASIL patients.

Data of sporadic SVD patients was obtained from the Radboud University Nijmegen Diffusion tensor and Magnetic resonance Cohort (RUN DMC). This prospective study recruited 503 non-demented elderly patients (aged 50 to 85 years) with SVD, defined as the presence of lacunes and/or WMH on neuroimaging. Patients were recruited in a hospital-based setting. Clinical examination and blood draw were performed on the same day, and the majority of patients underwent an MRI scan within 2 weeks. More details can be found in the previously published study protocol. ${ }^{9}$ Sixty-four subjects were excluded because of the presence of an old, large vessel territorial infarct $(n=55)$, insufficient MRI quality $(n=4)$, or missing clinical/neuropsychological data $(n=5)$. The final sporadic SVD sample comprised 439 patients.

In addition, healthy control subjects ( $n=93$ ) were drawn from our biobank repository, where we collected samples from healthy subjects across multiple studies. These subjects were relatives of outpatient clinic patients or volunteers recruited through advertisements. The absence of cerebrovascular events or other neurological symptoms/diagnoses was established through a clinical interview and neurological examination by a board-certified neurologist.

All measurements were performed blinded from each other. The two SVD samples were subjected to independent statistical analyses. The studies were approved by the ethics committee of the respective institution. Written and informed consent was obtained from all subjects after receiving a complete description of the study.

\section{Clinical characterization}

All SVD patients were characterized according to standardized procedures. Clinical severity was assessed using established scales for activities of daily living (Barthel scale), focal neurological deficits (National Institutes of Health stroke scale [NIHSS]), and disability (modified Rankin scale [mRS]). ${ }^{10}$ The latter two were only available in the CADASIL sample. 


\section{Neuropsychological testing}

Patients underwent comprehensive neuropsychological testing. In the current study, we focused on processing speed performance, the main cognitive deficit in SVD patients. ${ }^{11,12}$

For CADASIL patients, trail making tests (matrix A and B) were available. For sporadic SVD patients, the 1-letter subtask of the Paper-Pencil Memory Scanning Test and the Letter-Digit Substitution Task were available. For each test, age- and educationcorrected z-scores were first calculated in reference to a healthy control population from the literature. ${ }^{13-15}$ The mean of both $z$ scores was then used as compound processing speed z-score.

\section{Serum biobanking}

Blood samples were collected through a standardized procedure. Blood was drawn into serum containers with clotting activator and subsequently allowed to clot for at least $30 \mathrm{~min}$ utes at room temperature. Separation of serum was achieved by centrifugation at 2,000 $x g$ for 10 minutes. Samples were aliquoted in polypropylene screw cap vials and stored deep-frozen until analysis.

\section{NfL assay}

All samples were analyzed on the same single-molecule array instrument (Simoa HD-1, Quanterix, Lexington, MA, USA) in Basel. We used the capture monoclonal antibody (mAB) 47:3 and the biotinylated detector $m A B$ 2:1 (UmanDiagnostics, Umeå, Sweden), ${ }_{1}^{16}$ transferred onto the Simoa platform. Bovine lyophilized NfL was obtained from UmanDiagnostics. Calibrators ranged from 0 to $2,000 \mathrm{pg} / \mathrm{mL}$. Intra- and inter-assay variabilities were below $20 \%$. The analytical sensitivity was 0.32 $\mathrm{pg} / \mathrm{mL}$. All samples produced signals above the analytical sensitivity of the assay.

\section{MRI scanning and analysis}

Within the study, all SVD patients were examined on a single MRI scanner (CADASIL, 3 Tesla Siemens Magnetom Verio; sporadic SVD, 1.5 Tesla Siemens Magnetom Sonata, Siemens Healthcare, Erlangen, Germany) with a standardized protocol including 3D-T1, fluid-attenuated inversion recovery, $\mathrm{T} 2$, and DTI sequences. Sequence parameters have already been published. ${ }^{17}$ We calculated $\mathrm{WMH}$, lacune, and brain volumes and counted cerebral microbleeds as previously described..$^{17,18}$ For normalization, all volumes were divided by the volume of the intracranial cavity.

Mean diffusivity is an established measure for SVD burden calculated from DTI. It captures the microstructural integrity of white matter through diffusivity of water in the tissue. Diffusion images were first pre-processed to correct for eddy-cur- rent-induced distortions and motion as previously described. ${ }^{17}$ The diffusion tensor was estimated using "dtifit," part of the Functional Magnetic Resonance Imaging of the Brain (FMRIB) Software Library (FSL). ${ }^{19,20}$ To avoid contamination by CSF partial volume, the analysis of mean diffusivity was restricted to the main fiber tracts. For this purpose, diffusion data were skeletonized as implemented in the tract-based spatial statistics pipeline (TBSS) of FSL. ${ }^{21}$ In brief, all subjects' fractional anisotropy data were aligned into a common space using nonlinear registration and the standard fractional anisotropy template provided within the FSL. Each subject's fractional anisotropy data were then projected onto the tract skeleton in standard space. Finally, mean diffusivity images were projected onto the skeleton using the fractional-anisotropy-derived projection parameters. The final mean diffusivity skeletons were masked with the standard skeleton threshold value of 0.3 to focus the analysis on main fiber tracts. Furthermore, regions of the skeleton directly adjacent to the ventricles were removed by a custom-made mask to further minimize the contamination of the skeleton by CSF partial volume. Finally, the mean over the entire mean diffusivity skeleton was used for subsequent analyses.

\section{Statistical analysis}

Statistical analyses were performed in "R," version 3.1.2. ${ }^{22}$ For group comparisons, we used the nonparametric Wilcoxon rank sum test (with Bonferroni correction for multiple comparisons). The ability of serum NfL to discriminate between SVD patients and healthy controls was assessed using receiver operating characteristic analysis as implemented in the $\mathrm{R}$ package "ROCR" (version 1.0-7). ${ }^{23}$

We applied linear regression analysis to assess associations with serum NfL levels as well as processing speed compound scores as dependent variables. All $R^{2}$ values reported are "adjusted $R^{2}$." Variables were power transformed when necessary to ensure the appropriateness of linear models as indicated by the distribution of residuals. To ensure that regression results were robust and not driven by outliers, we conducted a statistical regression outlier test as implemented in the "car" package of "R."24 As a result, two CADASIL patients and two sporadic SVD patients had to be excluded from the regression analyses. In order to determine associations with clinical scores (NIHSS and mRS as dependent variables), we used ordinal logistic regression as implemented in the "R" package "ordinal."25 For all analyses, correction for multiple testing was performed via the Bonferroni method.

For multiple regression, we first applied the least absolute shrinkage and selection operator (lasso) regression for variable 
selection as implemented in the R package "glmnet" (for linear response) or "glmnetcr" (for ordinal response) with standard parameters. ${ }^{26}$ Lasso performs both variable selection and regularization in order to enhance the prediction accuracy and interpretability of regression models. Serum NfL, all MRI markers, age, and sex were included as independent variables in lasso regression, and variables with nonzero coefficients after crossvalidation were carried forward to the final multiple regression model.

\section{Results}

The characteristics of SVD patients and healthy controls are presented in Table 1. Patients in both SVD samples, CADASIL and sporadic SVD, were relatively mildly affected as indicated by unimpaired activities of daily living (Barthel scale). Healthy controls covered the entire age range of both SVD samples (30 to 85 years).

In both SVD samples, a substantial number of patients had suffered from a prior stroke or transient ischemic attack (TIA). Serum NfL levels in these patients were not higher than those in patients without history of a cerebrovascular event (CADASIL, $P=0.144$; sporadic SVD, $P=0.190$ ).

\section{Serum NfL level in SVD}

In comparison with healthy controls, we found increased serum NfL levels in both SVD samples (CADASIL, $P=4.1 \mathrm{e}-06$; sporadic $S V D, P<1 \mathrm{e}-15$, Wilcoxon rank sum tests with Bonferroni correction) (Figure 1A). These differences were also significant after correction for age (CADASIL, $P=4.2 \mathrm{e}-12$; sporadic SVD, $P<1$ e-15) (Supplementary Figure 1). The ability of serum NFL to differentiate between diagnostic groups as assessed by receiver

Table 1. Characteristics of the study samples

\begin{tabular}{|c|c|c|c|}
\hline Characteristic & $\begin{array}{l}\text { CADASIL (Munich) } \\
(n=53)\end{array}$ & $\begin{array}{l}\text { Sporadic SVD (RUN DMC) } \\
\qquad(n=439)\end{array}$ & $\begin{array}{l}\text { Healthy controls } \\
\qquad(n=93)\end{array}$ \\
\hline \multicolumn{4}{|l|}{ Demographic characteristics } \\
\hline Age (yr), median (IQR) & $56.0(11.2)$ & $64.3(15.2)$ & $59(12.6)$ \\
\hline Female sex, n (\%) & $36(67.9)$ & $199(45.3)$ & $58(62.4)$ \\
\hline Prior stroke/TIA, n (\%) & $28(52.8)$ & $162(36.9)$ & 0 \\
\hline Last stroke/TIA (yr), median (IOR) & $2.9(8.9)$ & $0.7(1.7)$ & NA \\
\hline \multicolumn{4}{|l|}{ Vascular risk factors, n (\%) } \\
\hline Hypertension & $11(20.8)$ & $316(72)$ & $16(17.2)$ \\
\hline Hypercholesterolemia & $24(45.3)$ & $193(44)$ & $12(12.9)$ \\
\hline Diabetes & $0(0)$ & $59(13.4)$ & $5(5.4)$ \\
\hline Current or past smoking & $34(64.2)$ & $304(69.2)$ & NA \\
\hline \multicolumn{4}{|l|}{ Clinical scores, median (IOR) } \\
\hline Barthel scale score & $100(0)$ & $100(0)$ & NA \\
\hline Processing speed z-score & $-0.56(2.21)$ & $-0.31(1.58)$ & NA \\
\hline NIHSS score & $0(1)$ & NA & NA \\
\hline $\mathrm{mRS}$ score & $0(1)$ & NA & NA \\
\hline \multicolumn{4}{|l|}{ SVD markers, median (IQR) } \\
\hline Serum NfL (pg/mL) & $41.7(46.9)$ & $50.8(38.9)$ & $26.0(14.7)$ \\
\hline WMH volume (\%)* & $7.52(7.39)$ & $0.59(1.23)$ & NA \\
\hline Lacune volume (\%)* & $0.024(0.064)$ & $0(0)$ & NA \\
\hline Microbleed count & $0(3)$ & $0(0)$ & NA \\
\hline Brain volume $(\%)^{*}$ & $78.4(6.82)$ & $65.5(7.69)$ & NA \\
\hline Mean diffusivity $\left(10^{-4} \mathrm{~mm}^{2} / \mathrm{sec}\right)$ & $9.86(1.83)$ & $8.02(0.55)$ & NA \\
\hline
\end{tabular}

Interquartile range (IQR) is defined as the difference between the 75th and 25th percentiles.

CADASIL, Cerebral Autosomal-Dominant Arteriopathy with Subcortical Infarcts and Leukoencephalopathy; SVD, small vessel disease; RUN DMC, Radboud University Nijmegen Diffusion tensor and Magnetic resonance Cohort; TIA, transient ischemic attack; NA, not available; NIHSS, National Institutes of Health stroke scale; mRS, modified Rankin scale; NfL, neurofilament light chain; WMH, white matter hyperintensity.

*Normalized to intracranial volume. 
operating characteristics is shown in Figure 1B.

In all groups, serum NfL was positively correlated with age (Table 2), but there was a significant interaction with the diag-
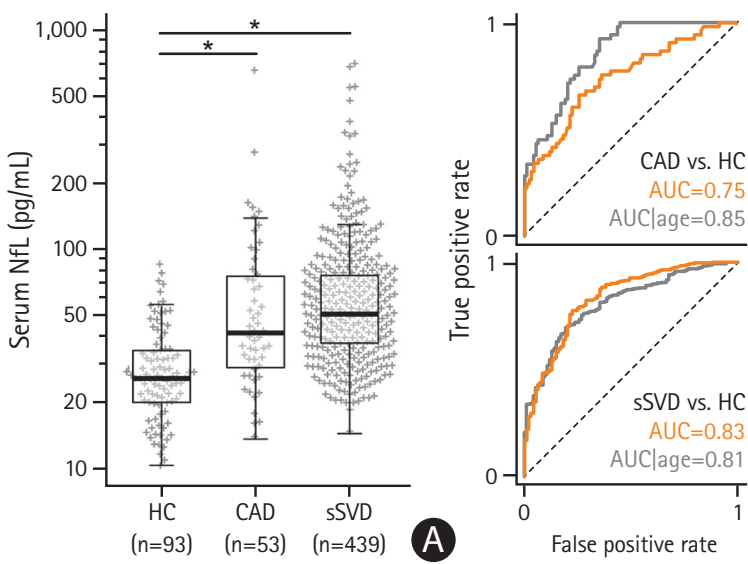

Figure 1. Increased serum neurofilament light chain (NfL) level in small vessel disease (SVD). (A) Compared with healthy controls (HCs), serum NfL levels are increased in CADASIL (CAD) and sporadic SVD (sSVD) patients. Please note the logarithmic scale. (B) Receiver operating characteristic analyses for serum NfL: CAD patients vs. HCs (top panel) and SSVD patients vs. HCs (bottom panel). Receiver operating characteristic curves were generated using raw serum NfL values (orange lines) and values after regressing out the effect of age (gray lines). CADASIL, Cerebral Autosomal-Dominant Arteriopathy with Subcortical Infarcts and Leukoencephalopathy; AUC, area under the curve; AUClage, AUC corrected for age. ${ }^{*} P<0.001$ (Wilcoxon rank sum test with Bonferroni correction). nosis: patients with sporadic SVD showed a steeper increase of serum NfL levels with age than healthy controls $(P=0.011$ for interaction).

\section{Serum NfL level and MRI markers for SVD}

We next assessed associations between serum NfL levels and established MRI markers for SVD as well as age and sex. Simple linear regression analyses (Table 2) showed significant associations between serum NfL and all imaging markers as well as age, in both the CADASIL and sporadic SVD samples. Regarding SVD imaging markers, the strongest effect was found for mean diffusivity in both samples. Most associations remained significant after controlling for age (Table 2).

Serum NfL level and processing speed impairment In order to investigate the association between serum NfL levels and cognitive deficits, we focused on processing speed performance, the cognitive domain most affected in SVD patients. ${ }^{11,12}$

In both samples, CADASIL and sporadic SVD, simple linear regression analysis showed a significant association between serum NfL levels and processing speed scores (Table 3 and Figure $2 \mathrm{~A}$ and $\mathrm{B}$ ). Significant associations were also found between all imaging markers and processing speed scores (Table 3). In both samples, mean diffusivity showed the strongest effect and was the only imaging parameter explaining more processing speed variance than serum NfL levels. The associa-

Table 2. Linear regression models with serum NfL levels as dependent variable

\begin{tabular}{|c|c|c|c|c|}
\hline & $\beta^{*}$ & $P$ & $P(\text { age })^{+}$ & $\mathrm{R}^{2}$ \\
\hline \multicolumn{5}{|l|}{ CADASIL } \\
\hline Mean diffusivity & 0.730 & $1.2 \mathrm{e}-09^{\ddagger}$ & $1.2 \mathrm{e}-05^{\dagger}$ & 0.52 \\
\hline Age & 0.629 & $7.8 \mathrm{e}-07^{\ddagger}$ & - & 0.38 \\
\hline Brain volume $^{\S}$ & -0.595 & $4.1 e-06^{\dagger}$ & $0.005^{\ddagger}$ & 0.34 \\
\hline WMH volume ${ }^{\S}$ & 0.575 & $1.0 \mathrm{e}-05^{\ddagger}$ & 0.023 & 0.32 \\
\hline Lacune volume ${ }^{\S}$ & 0.558 & $2.1 \mathrm{e}-05^{\ddagger}$ & $6.41 \mathrm{e}-04^{\ddagger}$ & 0.30 \\
\hline Microbleed count & 0.366 & 0.008 & 0.021 & 0.11 \\
\hline Sex & 0.117 & 0.418 & 0.825 & 0 \\
\hline \multicolumn{5}{|l|}{ Sporadic SVD } \\
\hline Age & 0.558 & $<1 \mathrm{e}-15^{\dagger}$ & - & 0.31 \\
\hline Mean diffusivity & 0.461 & $<1 \mathrm{e}-15^{\ddagger}$ & $7.31 \mathrm{e}-05^{\ddagger}$ & 0.21 \\
\hline WMH volume ${ }^{\S}$ & 0.382 & $<1 \mathrm{e}-15^{\ddagger}$ & $1.08 \mathrm{e}-05^{\neq}$ & 0.14 \\
\hline Brain volume $\mathrm{e}^{\S}$ & -0.376 & $<1 \mathrm{e}-15^{+}$ & 0.248 & 0.14 \\
\hline Lacune volume $\mathrm{e}^{\S}$ & 0.264 & $2.2 \mathrm{e}-08^{+}$ & $2.18 \mathrm{e}-06^{\dagger}$ & 0.07 \\
\hline Microbleed count & 0.149 & $0.0019^{\dagger}$ & 0.007 & 0.02 \\
\hline Sex & -0.003 & 0.947 & 0.993 & 0 \\
\hline
\end{tabular}

NfL, neurofilament light chain; CADASIL, Cerebral Autosomal-Dominant Arteriopathy with Subcortical Infarcts and Leukoencephalopathy; WMH, white matter hyperintensity; SVD, small vessel disease.

${ }^{*}$ Standardized $\beta ;{ }^{+}$Controlled for age, P given age; ${ }^{\ddagger}$ Significant after correction for multiple testing (Bonferroni); ${ }^{5}$ Normalized to intracranial volume. 
Table 3. Linear regression models with processing speed as dependent variable

\begin{tabular}{|c|c|c|c|}
\hline & $\beta^{*}$ & $P$ & $\mathrm{R}^{2}$ \\
\hline \multicolumn{4}{|c|}{ CADASIL: simple linear regression } \\
\hline Mean diffusivity & -0.626 & $9.0 \mathrm{e}-07^{+}$ & 0.38 \\
\hline Serum NfL & -0.526 & $7.6 \mathrm{e}-05^{+}$ & 0.27 \\
\hline Lacune volume $^{\dagger}$ & -0.521 & $9.0 \mathrm{e}-05^{+}$ & 0.26 \\
\hline Microbleed count & -0.486 & $3.0 \mathrm{e}-04^{+}$ & 0.22 \\
\hline WMH volume ${ }^{\ddagger}$ & -0.474 & $4.5 \mathrm{e}-04^{+}$ & 0.21 \\
\hline Brain volume $^{\neq}$ & 0.374 & 0.007 & 0.12 \\
\hline Age & -0.240 & 0.091 & 0.04 \\
\hline Sex & 0.019 & 0.895 & 0 \\
\hline \multicolumn{4}{|c|}{ CADASIL: multiple linear regression (lasso): $P=6.6 \mathrm{e}-07, \mathrm{R}^{2}=0.45$} \\
\hline Mean diffusivity & -0.340 & 0.022 & - \\
\hline Lacune volume & -0.283 & 0.029 & - \\
\hline Microbleed count & -0.256 & 0.041 & - \\
\hline \multicolumn{4}{|c|}{ Sporadic SVD: simple linear regression } \\
\hline Mean diffusivity & -0.267 & $1.5 \mathrm{e}-08^{+}$ & 0.07 \\
\hline Serum NfL & -0.257 & $4.8 \mathrm{e}-08^{+}$ & 0.06 \\
\hline WMH volume ${ }^{*}$ & -0.207 & $1.3 e-05^{+}$ & 0.04 \\
\hline Lacune volume $^{\ddagger}$ & -0.174 & $2.6 \mathrm{e}-04^{+}$ & 0.03 \\
\hline Brain volume $^{\ddagger}$ & 0.173 & $2.7 \mathrm{e}-04^{+}$ & 0.03 \\
\hline Age & -0.149 & $0.0018^{+}$ & 0.02 \\
\hline Microbleed count & -0.140 & $0.0035^{+}$ & 0.02 \\
\hline Sex & 0.105 & 0.0279 & 0.01 \\
\hline \multicolumn{4}{|c|}{ Sporadic SVD: multiple linear regression (lasso): $P=4.8 \mathrm{e}-10, R^{2}=0.09$} \\
\hline Mean diffusivity & -0.188 & $2.92 \mathrm{e}-04$ & - \\
\hline Serum NfL & -0.171 & $9.97 e-04$ & - \\
\hline
\end{tabular}

CADASIL, Cerebral Autosomal-Dominant Arteriopathy with Subcortical Infarcts and Leukoencephalopathy; NfL, neurofilament light chain; WMH, white matter hyperintensity; SVD, small vessel disease.

${ }^{*}$ Standardized $\beta$; ${ }^{+}$Simple regression significant after correction for multiple testing (Bonferroni); ${ }^{*}$ Normalized to intracranial volume.

tions between the other imaging markers and processing speed were weaker than for serum NfL.

In the CADASIL sample, the lasso regression model including serum NfL, all imaging markers, age, and sex as independent variables selected mean diffusivity, lacune volume, and microbleed count for the final model. In the sporadic SVD sample, lasso regression selected mean diffusivity and serum NfL (Table 3).

\section{Serum NfL level and neurological severity and functional disability}

Using the CADASIL sample, we were also able to assess the association between serum NfL levels and clinical scores capturing focal neurological deficits (NIHSS) and disability (mRS).

For NIHSS, simple ordinal logistic regression showed the strongest association with serum NfL levels (Table 4 and Figure $2 \mathrm{C}$ ). Significant, albeit weaker, associations were also found between imaging markers (except for microbleeds) and NIHSS scores. After ordinal lasso regression including serum NfL levels, all imaging markers, age, and sex as independent variables, the final model comprised mean diffusivity, brain volume, and serum NfL (Table 4).

For disability, simple ordinal logistic regression showed the most significant association between serum NfL levels and mRS scores (Table 4 and Figure 2D), and associations between all imaging markers and $\mathrm{mRS}$ scores were weaker. The final model after lasso regression comprised serum NfL, brain volume, and (at trend level) microbleed count and mean diffusivity (Table 4).

\section{Discussion}

Analyzing two independent SVD samples, we found elevated serum NfL levels compared with healthy controls. Serum NfL 


\section{CADASIL}
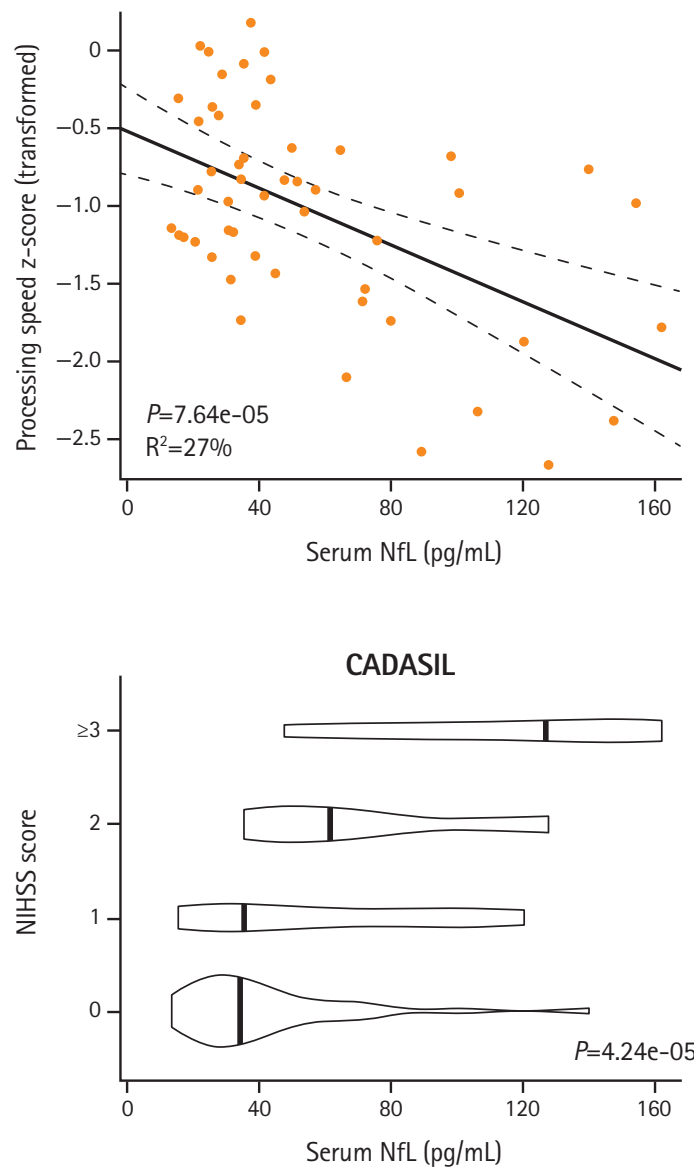
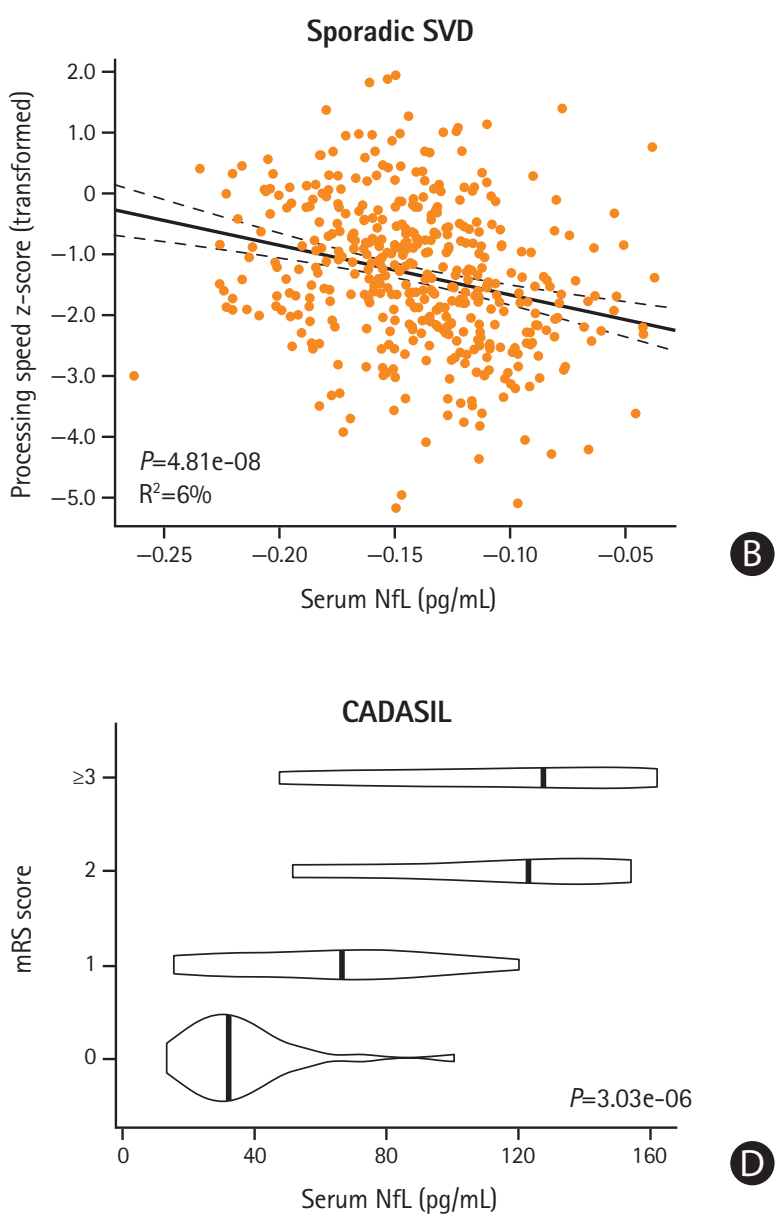

Figure 2. Serum neurofilament light chain (NfL) levels were associated with clinical deficits. (A, B) Simple linear regression analysis between serum NfL levels and processing speed in the Cerebral Autosomal-Dominant Arteriopathy with Subcortical Infarcts and Leukoencephalopathy (CADASIL) (A) and sporadic small vessel disease (SVD) (B) sample. Dashed lines depict the 95\% confidence interval for the linear regression. (C, D) In the CADASIL sample, logistic regression analysis showed an independent association between serum NfL levels and National Institutes of Health stroke scale (NIHSS) scores (C) as well as modified Rankin scale (mRS) scores (D). Associations are visualized using violin plots. Vertical lines depict the median serum NfL levels in each score category. For both scores, values of 3 and 4 were grouped together for visualization because of the low number of patients in these categories.

levels were associated with all MRI markers of SVD burden, in particular mean diffusivity. Regarding clinical symptoms, serum $\mathrm{NfL}$ levels were strongly associated with impaired processing speed performance, the cognitive domain most prominently affected in SVD. These findings were consistent across young patients with genetically defined SVD (CADASIL) and patients with sporadic SVD. In CADASIL patients, serum NfL levels were also strongly and independently associated with measures of focal neurological deficits and disability.

Elevated serum NfL is not specific for a particular pathology, as it is released upon neuroaxonal damage of any cause. Recent findings indicate a role of serum NfL in multiple sclerosis $_{1}{ }^{27}$ motor neuron disease, ${ }^{1}$ and-particularly important in the elderly population-neurodegenerative disorders, ${ }^{28}$ i.e., $\mathrm{Al}-$ zheimer's disease ${ }^{2}$ and frontotemporal dementia. ${ }^{3}$ Our findings demonstrate that SVD burden is a relevant contributor to ele- vated serum NfL levels. The increase of serum NfL levels in SVD compared with healthy controls (approximately two-fold on average) is equal if not higher than the increase seen for patients with Alzheimer's disease. ${ }^{2,28}$ The association between SVD burden and serum NfL together with the high prevalence of SVD suggests that SVD needs to be considered when interpreting serum NfL levels in elderly patients.

Our results potentially extend the utility of serum NfL as a marker for SVD burden. To our knowledge, our analysis of serum NfL is the first study to identify a blood-based marker associated with both imaging and clinical features of SVD. For processing speed performance in the sporadic SVD sample, there was an added value of serum NfL levels beyond MRI markers. Moreover, in both samples, only mean diffusivity explained more variance in processing speed than serum NfL. All other imaging markers of SVD-i.e., WMH, lacune, or brain vol- 
Table 4. Logistic regression models with NIHSS and mRS scores as dependent variables in the CADASIL sample

\begin{tabular}{|c|c|c|c|}
\hline Variable & $\mathrm{OR}^{*}$ & $95 \% \mathrm{Cl}^{+}$ & $P$ \\
\hline \multicolumn{4}{|c|}{ NIHSS score: simple ordinal logistic regression } \\
\hline Serum NfL & 4.11 & $2.16-8.47$ & $4.2 \mathrm{e}-05^{\dagger}$ \\
\hline Mean diffusivity & 3.67 & $1.93-7.67$ & $1.8 \mathrm{e}-04^{+}$ \\
\hline Lacune volume $e^{\S}$ & 2.99 & $1.68-5.80$ & $4.3 e-04^{+}$ \\
\hline Brain volume ${ }^{\S}$ & 0.26 & $0.11-0.53$ & $6.6 \mathrm{e}-04^{\dagger}$ \\
\hline WMH volume ${ }^{\S}$ & 2.53 & $1.38-4.96$ & $0.004^{\ddagger}$ \\
\hline Age & 1.88 & $0.99-4.01$ & 0.069 \\
\hline Microbleed count & 1.41 & $0.89-2.36$ & 0.121 \\
\hline Sex & 1.39 & $0.79-5.43$ & 0.245 \\
\hline \multicolumn{4}{|c|}{ NIHSS score: multiple ordinal logistic regression (lasso) } \\
\hline Mean diffusivity & 3.27 & $1.23-9.53$ & 0.021 \\
\hline Brain volume $^{\S}$ & 0.33 & $0.11-0.86$ & 0.030 \\
\hline Serum NfL & 2.51 & $1.10-6.08$ & 0.032 \\
\hline \multicolumn{4}{|c|}{ mRS score: simple ordinal logistic regression } \\
\hline Serum NfL & 5.52 & $2.83-12.0$ & $3.0 \mathrm{e}-06^{\dagger}$ \\
\hline Mean diffusivity & 5.97 & $2.84-14.4$ & $1.2 \mathrm{e}-05^{+}$ \\
\hline Brain volume $^{\S}$ & 0.20 & $0.08-0.41$ & 7.0e- $-05^{+}$ \\
\hline WMH volume ${ }^{\S}$ & 3.47 & $1.78-7.40$ & $5.5 \mathrm{e}-04^{\ddagger}$ \\
\hline Lacune volume ${ }^{\S}$ & 2.71 & $1.57-5.10$ & 7.7e- $-04^{+}$ \\
\hline Microbleed count & 6.12 & $2.17-35.9$ & 0.008 \\
\hline Age & 2.52 & $1.33-5.45$ & 0.009 \\
\hline Sex & 1.57 & $0.92-2.72$ & 0.097 \\
\hline \multicolumn{4}{|c|}{ mRS score: multiple ordinal logistic regression (lasso) } \\
\hline Serum NfL & 2.65 & $1.13-6.63$ & 0.028 \\
\hline Brain volume $^{\S}$ & 0.38 & $0.15-0.87$ & 0.029 \\
\hline Microbleed count & 2.35 & $0.99-9.29$ & 0.103 \\
\hline Mean diffusivity & 2.14 & $0.75-6.21$ & 0.151 \\
\hline
\end{tabular}

NIHSS, National Institutes of Health stroke scale; mRS, modified Rankin scale; CADASIL, Cerebral Autosomal-Dominant Arteriopathy with Subcortical Infarcts and Leukoencephalopathy; $\mathrm{OR}$, odds ratio; $\mathrm{Cl}$, confidence interval; $\mathrm{NfL}$, neurofilament light chain; $\mathrm{WMH}$, white matter hyperintensity.

${ }^{*}$ Cumulative $0 \mathrm{R} ;{ }^{+} 95 \% \mathrm{Cl}$ for the cumulative $\mathrm{OR} ;{ }^{*}$ Simple regression significant after correction for multiple testing (Bonferroni); ${ }^{5}$ Normalized to intracranial volume.

ume-showed a weaker association with processing speed, suggesting that serum NfL might be of equal or even greater utility than these conventional MRI markers.

The strong relationship between DTI measures and vascular cognitive impairment is well established, both in cross-sectional and longitudinal studies. ${ }^{17,29,30}$ DTI quantifies alterations in the microstructural integrity of the brain tissue, and this seems to be a major substrate underlying cognitive impairment in SVD. ${ }^{31}$ Interestingly, among imaging markers, serum NfL levels showed the strongest association with mean diffusivity, suggesting that the two parameters capture a similar disease process: NfL is regarded as a marker for neuroaxonal damage, and damage to axons is also a potential explanation for mean diffusivity changes in the white matter. The high intercorrelation might also explain why the association between serum NfL and processing speed was not independent from mean diffusivity in the CADASIL sample.

A recent study showed that serum NfL is sensitive to active $S V D$, i.e., recent subcortical infarcts, and that serum NfL levels remain elevated at least 3 months after the acute infarct. ${ }^{5}$ We did not find a difference in serum NfL levels between patients with and without history of stroke or TIA. However, by design, all of the events in CADASIL patients occurred more than 3 months ago. In the same study, serum NfL levels were especially high in patients with incident SVD lesions on follow-up scans. Adding to that study, our results suggest that serum NfL levels also reflect the chronic SVD burden, as, e.g., captured by mean diffusivity, as well as the clinical severity. 
MRI will remain the gold standard for assessment of SVD burden. Imaging markers have already been refined to serve as surrogate treatment response markers for clinical trials in SVD. ${ }^{17,29,32}$ However, there are relevant limitations of MRI in the context of clinical routine and trials. First, some patients cannot be assessed because of contraindications (e.g., metal implants) or claustrophobia. Second, data quality can be affected by disease severity, therefore leading to bias. For example, cognitively impaired patients are more likely to cause motion artifacts. ${ }^{33}$ Third, MRI is relatively expensive and time consuming. This applies to both the examination itself and the mandatory post-processing for assessing SVD burden. ${ }^{34}$ Finally, even after protocol harmonization, MRI is prone to center effects in multicenter studies, introduced by inevitable differences in MRI scanner hardware and software. ${ }^{35}$ In contrast to MRI, blood draws are less costly and less time consuming, can be readily repeated at multiple time points in longitudinal studies, and are less prone to bias. Furthermore, for multicenter studies, blood biomarkers can be centrally analyzed at a single, experienced (reference) laboratory. Serum NfL might therefore complement MRI measures in assessing disease burden in SVD patients.

Several other blood biomarkers have been studied in SVD, such as markers for inflammation, endothelial dysfunction, subclinical cardiac injury, and coagulation. Results on these markers have so far been ambiguous: while, in some studies, associations with imaging parameters, such as WMH, have been reported ${ }_{1}^{36-40}$ this was not the case in others. ${ }^{41,42}$ Most importantly, in contrast to our present findings for NfL, no other blood marker has been reported to have robust associations with both imaging and clinical features of SVD. Although NfL is not specific for SVD, our results suggest that serum NfL may be used to stage disease severity. It could potentially also be used to monitor the disease progression, but this needs to be tested in longitudinal studies. Ultimately, serum NfL might be used as an outcome parameter to assess treatment effects in clinical trials.

Further, in our study, serum NfL levels were associated with age, a phenomenon already observed in some, ${ }^{1,28}$ but not all, previous studies. ${ }^{43,44}$ Given the age-independent associations between serum NfL and MRI markers as well as clinical measures, serum NfL levels in our patients with hereditary SVD are most likely reflecting disease progression with age rather than a pure age effect. For the sporadic SVD sample, an even stronger age effect was observed, which might reflect concomitant age-related disease processes, such as neurodegenerative pathology.

A limitation of our study is its cross-sectional design, not yet allowing longitudinal analyses in regard to the prognostic value of serum NfL levels in SVD patients. The lack of neuroimaging in healthy controls can also be considered a limitation, as we were not able to exclude subclinical SVD or other covert brain diseases in these subjects. Presence of brain pathology leading to neuroaxonal damage and therefore elevated serum NfL levels in controls could have led to an underestimation of the difference between controls and SVD patients. Another limitation is the lack of NIHSS and mRS scores in the sporadic SVD sample. The main strengths of our study are the inclusion of patients with genetically defined SVD, thus limiting potential confounding factors, and the independent validation of a large sample of patients with sporadic SVD. All SVD subjects received comprehensive and standardized image acquisition and post-processing, including DTI and quantitative volumetric analysis, as well as serum sampling according to standard operating procedures. Serum NfL was quantified at a single reference laboratory using a state-of-the-art, ${ }_{1}^{45}$ highly sensitive Simoa assay.

\section{Conclusions}

SVD burden was reflected in serum NfL levels and needs to be considered when interpreting elevated NfL levels in elderly patients. Serum NfL was associated with imaging as well as clinical features in hereditary and sporadic SVD and might therefore complement established MRI markers in assessing SVD burden. Since neurodegenerative pathology and SVD often cooccur in elderly patients, serum NfL might offer the possibility of assessing the combined effect of these pathologies on brain integrity. Serum NfL might also be a promising candidate marker for prognostication and treatment response monitoring, which needs to be addressed in longitudinal studies.

\section{Supplementary materials}

Supplementary materials related to this article can be found online at https://doi.org/10.5853/jos.2017.02565.

\section{Disclosure}

The authors have no financial conflicts of interest.

\section{Acknowledgments}

This study has been funded by the LMU FöFoLe program (RegNr. 808), Else Kröner-Fresenius-Stiftung (2014_A200), Alzheimer Forschung Initiative e.V. (\#16018CB), Neurology Research Pool (University Hospital Basel), and Vascular Dementia Research Foundation. 


\section{References}

1. Lu CH, Macdonald-Wallis C, Gray E, Pearce N, Petzold A, Norgren $N$, et al. Neurofilament light chain: a prognostic biomarker in amyotrophic lateral sclerosis. Neurology 2015;84:22472257.

2. Mattsson N, Andreasson U, Zetterberg H, Blennow K; Alzheimer's Disease Neuroimaging Initiative. Association of plasma neurofilament light with neurodegeneration in patients with alzheimer disease. JAMA Neurol 2017;74:557-566.

3. Rohrer JD, Woollacott IO, Dick KM, Brotherhood E, Gordon E, Fellows $A$, et al. Serum neurofilament light chain protein is a measure of disease intensity in frontotemporal dementia. Neurology 2016;87:1329-1336.

4. Pantoni L. Cerebral small vessel disease: from pathogenesis and clinical characteristics to therapeutic challenges. Lancet Neurol 2010;9:689-701.

5. Gattringer $T$, Pinter $D$, Enzinger $C$, Seifert-Held $T$, Kneihsl $M$, Fandler $\mathrm{S}$, et al. Serum neurofilament light is sensitive to active cerebral small vessel disease. Neurology 2017;89:21082114.

6. Traenka C, Disanto G, Seiffge DJ, Gensicke H, Hert L, GrondGinsbach $C_{1}$ et al. Serum neurofilament light chain levels are associated with clinical characteristics and outcome in patients with cervical artery dissection. Cerebrovasc Dis 2015;40:222227.

7. Jonsson $M$, Zetterberg $H_{\text {, van Straaten }}$, Lind $K$, Syversen $S$, Edman $A$, et al. Cerebrospinal fluid biomarkers of white matter lesions: cross-sectional results from the LADIS study. Eur J Neurol 2010;17:377-382.

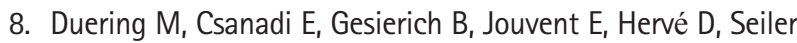
$S$, et al. Incident lacunes preferentially localize to the edge of white matter hyperintensities: insights into the pathophysiology of cerebral small vessel disease. Brain 2013;136(Pt 9):2717-2726.

9. van Norden $A G$, de Laat $K F$, Gons RA, van Uden IW, van Dijk $E J$, van Oudheusden $\sqcup$, et al. Causes and consequences of cerebral small vessel disease. The RUN DMC study: a prospective cohort study. Study rationale and protocol. BMC Neurol 2011;11:29.

10. Harrison JK, McArthur KS, Quinn TJ. Assessment scales in stroke: clinimetric and clinical considerations. Clin Interv Aging 2013;8:201-211.

11. Zieren $N$, Duering $M$, Peters $N$, Reyes $S$, Jouvent $E$, Hervé $D$, et al. Education modifies the relation of vascular pathology to cognitive function: cognitive reserve in cerebral autosomal dominant arteriopathy with subcortical infarcts and leukoencephalopathy. Neurobiol Aging 2013;34:400-407.
12. Charlton RA, Morris RG, Nitkunan A, Markus HS. The cognitive profiles of CADASIL and sporadic small vessel disease. Neurology 2006;66:1523-1526.

13. Tombaugh TN. Trail Making Test A and B: normative data stratified by age and education. Arch Clin Neuropsychol 2004; 19:203-214.

14. Van Der Elst W, Van Boxtel MP, Van Breukelen GJ, Jolles J. Assessment of information processing in working memory in applied settings: the paper and pencil memory scanning test. Psychol Med 2007;37:1335-1344.

15. Van der Elst W, Van Boxtel MP, Van Breukelen GJ, Jolles J. The Concept Shifting Test: adult normative data. Psychol Assess 2006;18:424-432.

16. Norgren N, Karlsson JE, Rosengren L, Stigbrand T. Monoclonal antibodies selective for low molecular weight neurofilaments. Hybrid Hybridomics 2002;21:53-59.

17. Baykara E, Gesierich B, Adam R, Tuladhar AM, Biesbroek JM,

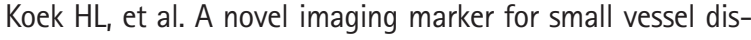
ease based on skeletonization of white matter tracts and diffusion histograms. Ann Neurol 2016;80:581-592.

18. Wollenweber FA, Baykara $E_{1}$ Zedde M, Gesierich $B$, Achmüller $M$, Jouvent $E$, et al. Cortical superficial siderosis in different types of cerebral small vessel disease. Stroke 2017;48:14041407.

19. Behrens TE, Berg HJ, Jbabdi S, Rushworth MF, Woolrich MW. Probabilistic diffusion tractography with multiple fibre orientations: what can we gain? Neuroimage 2007;34:144-155.

20. Behrens TE, Woolrich MW, Jenkinson $M$, Johansen-Berg $H$, Nunes RG, Clare $S$, et al. Characterization and propagation of uncertainty in diffusion-weighted MR imaging. Magn Reson Med 2003;50:1077-1088.

21. Smith $S M$, Jenkinson $M_{1}$ Johansen-Berg $H_{1}$ Rueckert $D$, Nichols $T E$, Mackay $C E$, et al. Tract-based spatial statistics: voxelwise analysis of multi-subject diffusion data. Neuroimage 2006;31: 1487-1505.

22. R Core Team. A Language and Environment for Statistical Computing. Vienna, AU: R Foundation for Statistical Computing, 2016.

23. Sing T, Sander O, Beerenwinkel N, Lengauer T. ROCR: visualizing classifier performance in R. Bioinformatics 2005;21:39403941.

24. Williams DA. Generalized linear model diagnostics using the deviance and single case deletions. J R Stat Soc Ser C Appl Stat 1987;36:181-191.

25. Christensen RH. Ordinal: regression models for ordinal data. http://cran.r-project.org/package=ordinal/. 2015. Accessed February 8, 2018.

26. Archer KJ. GImnetcr: fit a penalized constrained continuation 
ratio model for predicting an ordinal response. https://cran.rproject.org/package=glmnetcr. 2017. Accessed February 8, 2018.

27. Kuhle J, Plattner K, Bestwick JP, Lindberg RL, Ramagopalan SV, Norgren N, et al. A comparative study of CSF neurofilament light and heavy chain protein in MS. Mult Scler2013;19:15971603.

28. Gaiottino J, Norgren N, Dobson R, Topping J, Nissim A, Malaspina $A$, et al. Increased neurofilament light chain blood levels in neurodegenerative neurological diseases. PLoS One 2013;8:e75091.

29. Benjamin $P$, Zeestraten $E$, Lambert $C$, Ster IC, Williams $O A$, Lawrence $A$, et al. Progression of MRI markers in cerebral small vessel disease: sample size considerations for clinical trials. J Cereb Blood Flow Metab 2016;36:228-240.

30. de Laat KF, van Norden AG, Gons RA, van Oudheusden $\sqcup$, van Uden IW, Norris DG, et al. Diffusion tensor imaging and gait in elderly persons with cerebral small vessel disease. Stroke 2011;42:373-379.

31. Voineskos AN, Rajji TK, Lobaugh NJ, Miranda D, Shenton ME, Kennedy JL, et al. Age-related decline in white matter tract integrity and cognitive performance: a DTI tractography and structural equation modeling study. Neurobiol Aging 2012;33:21-34.

32. Pearce LA, McClure LA, Anderson DC, Jacova C, Sharma M, Hart RG, et al. Effects of long-term blood pressure lowering and dual antiplatelet treatment on cognitive function in patients with recent lacunar stroke: a secondary analysis from the SPS3 randomised trial. Lancet Neurol 2014;13:1177-1185.

33. Haller S, Monsch AU, Richiardi J, Barkhof F, Kressig RW, Radue EW. Head motion parameters in fMRI differ between patients with mild cognitive impairment and Alzheimer disease versus elderly control subjects. Brain Topogr 2014;27:801-807.

34. Wardlaw JM, Smith EE, Biessels GJ, Cordonnier C, Fazekas F, Frayne $R$, et al. Neuroimaging standards for research into small vessel disease and its contribution to ageing and neurodegeneration. Lancet Neurol 2013;12:822-838.

35. De Guio F, Jouvent E, Biessels GJ, Black SE, Brayne C, Chen C, et al. Reproducibility and variability of quantitative magnetic resonance imaging markers in cerebral small vessel disease. $J$ Cereb Blood Flow Metab 2016;36:1319-1337.

36. Nagai M, Hoshide $\mathrm{S}$, Kario K. Association of prothrombotic status with markers of cerebral small vessel disease in elderly hypertensive patients. Am J Hypertens 2012;25:1088-1094.

37. Satizabal CL, Zhu YC, Mazoyer B, Dufouil C, Tzourio C. Circulating IL-6 and CRP are associated with MRI findings in the elderly: the 3C-Dijon Study. Neurology 2012;78:720-727.

38. van Dijk EJ, Prins ND, Vermeer SE, Vrooman HA, Hofman A, Koudstaal PJ, et al. C-reactive protein and cerebral small-vessel disease: the Rotterdam Scan Study. Circulation 2005;112:900905.

39. Markus HS, Hunt B, Palmer K, Enzinger C, Schmidt $H$, Schmidt R. Markers of endothelial and hemostatic activation and progression of cerebral white matter hyperintensities: longitudinal results of the Austrian Stroke Prevention Study. Stroke 2005;36:1410-1414.

40. Vilar-Bergua A, Riba-Llena I, Penalba A, Cruz LM, JiménezBalado J, Montaner J, et al. $\mathrm{N}$-terminal pro-brain natriuretic peptide and subclinical brain small vessel disease. Neurology 2016;87:2533-2539.

41. Wiseman SJ, Doubal FN, Chappell FM, Valdés-Hernández MC, Wang $X$, Rumley $A$, et al. Plasma biomarkers of inflammation, endothelial function and hemostasis in cerebral small vessel disease. Cerebrovasc Dis 2015;40:157-164.

42. Schmidt R, Schmidt H, Pichler M, Enzinger C, Petrovic K, Niederkorn $\mathrm{K}$, et al. C-reactive protein, carotid atherosclerosis, and cerebral small-vessel disease: results of the Austrian Stroke Prevention Study. Stroke 2006;37:2910-2916.

43. Disanto G, Adiutori R, Dobson R, Martinelli V, Dalla Costa G, Runia $T$, et al. Serum neurofilament light chain levels are increased in patients with a clinically isolated syndrome. $J$ Neurol Neurosurg Psychiatry 2016;87:126-129.

44. Kuhle J, Barro C, Disanto G, Mathias A, Soneson C, Bonnier G, et al. Serum neurofilament light chain in early relapsing remitting MS is increased and correlates with CSF levels and with MRI measures of disease severity. Mult Scler 2016;22:15501559.

45. Kuhle J, Barro C, Andreasson U, Derfuss T, Lindberg R, Sandelius $\AA$, et al. Comparison of three analytical platforms for quantification of the neurofilament light chain in blood samples: ELISA, electrochemiluminescence immunoassay and Simoa. Clin Chem Lab Med 2016;54:1655-1661. 

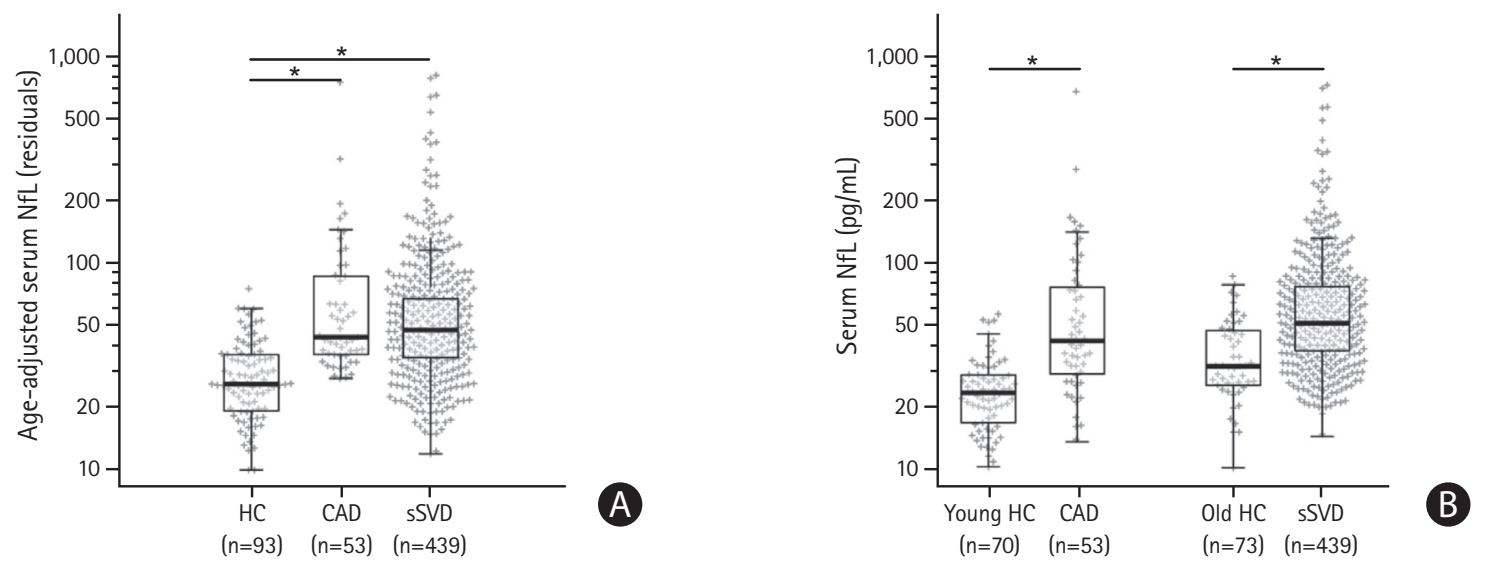

Supplementary Figure 1. Age-adjusted comparisons. (A) To account for age differences between the study samples, we calculated age-adjusted serum neurofilament light chain (NfL) levels (residuals after regressing out the effect of age). Compared with healthy controls (HCs), age-adjusted serum NfL levels were increased in CADASIL (CAD) and sporadic small vessel disease (SSVD) patients. (B) Comparison between serum NfL levels after matching HC subgroups for age (using similar median and interquartile range) to either the CADASIL (young $\mathrm{HC}$ ) or SSVD (old HC) sample. Please note the logarithmic scale in both panels. CADASIL, Cerebral Autosomal-Dominant Arteriopathy with Subcortical Infarcts and Leukoencephalopathy. ${ }^{*} P<0.001$ (Wilcoxon rank sum test with Bonferroni correction). 